\title{
Single Molecule DNA Biophysics with Atomic Force Microscopy
}

\author{
Dario Anselmetti ${ }^{\text {a), }}$ Jürgen Fritz ${ }^{\text {b) }}$, Benjamin Smith ${ }^{\text {c) }}$, \\ and Xavier Fernandez-Busquets \\ d)
}

a) Solvias AG, Physical Chemistry, CH-4002 Basel, Switzerland

b) IBM Zurich Research Laboratory, CH-8803 Rüschlikon, Switzerland and University of Basel, Institute of Physics, CH-4056 Basel, Switzerland

c) McGill University, Physics Department, Montreal (Quebec) H3A 2T8, Canada

d) University of Barcelona, Department of Biochemistry and Molecular Biology, E-08028 Barcelona, Spain

Correspondence to

PD Dr. Dario Anselmetti

Solvias AG, Klybeckstrasse 191

$\mathrm{CH}-4002$ Basel, Switzerland

Phone +41616866216

Fax +41616866096

e-mail dario.anselmetti@solvias.com

submitted 12 Mar 1999

published 31 Mar 2000

\begin{abstract}
Structural and functional properties of double stranded deoxyribonucleic acid (dsDNA) are investigated by atomic force microscopy (AFM) on a single molecule level. Here, we characterize different linear and circular DNA systems in terms of their geometry and topology, and visualize enzyme binding of restriction endonuclease Hae III to DNA. Manipulation of single DNA molecules is demonstrated by dissecting individual DNA strands. Furthermore, the elastic response of single DNA molecules to an externally applied force is investigated by AFM force spectroscopy experiments. This gives information about structural
\end{abstract}

properties of the DNA double helix. Specifically, transition from B-form to S-form DNA and a melting transition from double stranded to single stranded DNA is observed. This allows monitoring of specific interaction and binding of small intercalator molecules such as ethidium bromide $(\mathrm{EtBr})$ to DNA by means of a mechanical, non-fluorescent detection scheme.

\section{Introduction}

The observation and manipulation of nanometer sized objects, single molecules or individual atoms are the playground for nanoscience and nanotechnology [1]. While the classical macroscopic techniques normally rely on molecular ensemble experiments, which average over all possible molecular conformations and mutations, single molecule techniques (SMT) allow addressing and observation of molecular individuals in their individual conformation.

DNA plays an important role in many cellular processes like replication, homologous recombination, and transcription. Besides its genomic information, DNA exhibits very interesting biophysical and physicochemical properties which are essential for a proper functioning of the biomolecular processes involved. Fundamental phenomena like molecular elasticity, binding to proteins, supercoiling and electronic conductivity also depend on the numerous possible DNA conformations and can be investigated nowadays on a single molecule level. Experiments with single DNA molecules were reported with scanning tunneling microscopy [2], fluorescence microscopy [3], fluorescence correlation spectroscopy [4], optical tweezers [5, 6], bead techniques in magnetic fields [7, 8], optical microfibers [9], low energy electron point sources (electron holography) [10] 
and atomic force microscopy (AFM) [11]. All these methods provide directly or indirectly information on molecular structure and function. They differ, however, in the molecular properties they probe, their spatial and temporal resolution, their molecular sensitivity, and working environment.

AFM allows investigation of structural as well as functional properties of native biomolecules in liquid environments by a unique combination of subnanometer spatial resolution, millisecond temporal resolution and piconewton force sensitivity. Over the last decade, AFM investigations of single DNA molecules proved the successful concept of this microscopical single molecule technique. Additionally, AFM force spectroscopy experiments were conceived and applied to measure intermolecular interaction between receptor-ligands [12], antibody-antigens [13, 14], cell adhesion proteoglycans [15], oligonucleotides $[16,17]$ as well as single molecule reaction kinetics in cell adhesion molecules [18]. Furthermore, intramolecular elasticity phenomena like biopolymer structural transitions (dextran, DNA) [19, 20] and protein unfolding [21] were investigated giving access to mechanical properties of biomolecules and their related physiological phenomena.

In this work we focus on single molecule experiments with DNA by using different AFM modes of operation which not only allow observation but also manipulation of individual DNA molecules. Imaging of DNA by AFM reveals direct information on the topology of a DNA molecule and gives insights into its specific interaction with proteins like restriction endonuclease Hae III. Furthermore, we manipulate single DNA molecules by using the sharp tip of an AFM cantilever to dissect a distinct single DNA molecule. Finally, we demonstrate by AFM force spectroscopy how the elastic properties of single DNA molecules are varied by the absence/presence of small intercalator molecules such as ethidium bromide $(\mathrm{EtBr})$.

\section{Experimental}

\section{Biochemicals}

For the present study, the following biochemicals and chemicals were used: bacteriophage $\lambda$-DNA ( $\lambda$-DNA) from Boehringer-Roche (Basel, Switzerland), pGEM(-3Z) plasmid from Promega (Madison, Wisconsin, USA), supercoiled DNA ladder from Life Technologies (Basel, Switzerland), duplex poly(dG-dC) from Amersham Pharmacia Biotech AB (Uppsala, Sweden), Hae III restriction endonuclease from Boehringer-Roche (Basel, Switzerland), ethidium bromide (EtBr) from Fluka (Buchs, Switzerland) (see data sheet for safety precautions), and 3-aminopropyltriethoxysilane (APTES) from Fluka.

\section{Sample Preparation}

For DNA immobilization, APTES-functionalized mica [22] was incubated for 10 minutes with $1 \mu \mathrm{l}$ of $10 \mathrm{ng} / \mu \mathrm{l}$ DNA in TRIS$\mathrm{HCl}$ buffer (10 mM, pH 8.3). Afterwards, the mica was thoroughly rinsed with $1 \mathrm{ml}$ of nanopure water (Millipore, 18.2 $\mathrm{M} \Omega$ ) and dried in a dry nitrogen flow. For visualization of $\lambda$-DNA-Hae III complexes, an aliquot of Hae III restriction endonuclease $(1 \mathrm{u} / \mu \mathrm{l})$ was incubated with $\lambda$-DNA $(25 \mathrm{ng} / \mu \mathrm{l})$ for 2 hours in low-salt buffer at 4_C (10 mM TRIS-HCl, 10 $\mathrm{mM} \mathrm{MgCl}_{2}, 50 \mathrm{mM} \mathrm{NaCl}, 1 \mathrm{mM}$ Dithioerythritol (DTE), $\mathrm{pH}$ 7.5). Their consecutive immobilization on amino-terminated mica was identical as that described above. For AFM force spectroscopy experiments, poly(dG-dC) DNA $(1 \mu \mathrm{g} / \mu \mathrm{l})$ in TRIS- $\mathrm{HCl}$ buffer $(10 \mathrm{mM}, \mathrm{pH}$ 8.3) was immobilized on a freshly evaporated gold surface (50 nm Au / $2 \mathrm{~nm} \mathrm{Cr} /$ glass coverslip) by allowing a droplet of the solution to dry. The sample was rinsed with nanopure water to remove excess salt and mounted in the liquid cell filled with TRIS$\mathrm{HCl}$ buffer. EtBr was directly injected in the AFM liquid cell at a concentration of $10 \mu \mathrm{M}$ TRIS-HCl $(10 \mathrm{mM}, \mathrm{pH} 8.3)$.

\section{Atomic Force Microscopy}

All AFM data were taken with a commercial instrument (NanoScope IIIa - Multimode AFM, Veeco - Digital Instruments, USA). DNA molecules were imaged and dissected under ambient conditions with AFM silicon cantilevers (Nanoprobe, Wetzlar, Germany) in tapping mode of operation. Force spectroscopy experiments with single DNA molecules were performed in buffer solution (TRIS-HCl, $10 \mathrm{mM}, \mathrm{pH}$ 8.3) with standard silicon nitride cantilevers in a standard AFM liquid cell. Force distance curves were acquired with a point density of about 32000 per scan at a z-scan rate of typically $1 \mathrm{~Hz}$. The spring constants of the AFM cantilevers were determined within an estimated absolute error of $5 \%$ by two independent methods. In the first method, the spring constant was calculated upon using a mechanistic model based on geometrical and material considerations [23]. In the second method, the fluctuating cantilever deflections were frequency analyzed (power spectral density) yielding the time-averaged mechanical energy of the harmonic system, which was related to its thermal energy according to the equipartition principle [24].

\section{Data Analysis}

The AFM force spectroscopy plots were first corrected for cantilever bending to render real molecular extensions. In addition, we applied a box smoothing algorithm (window size $=10$ points) to reduce the experimental noise. Then the dataset was fitted using a polymer elasticity model (modified freely jointed chain model [5]). 

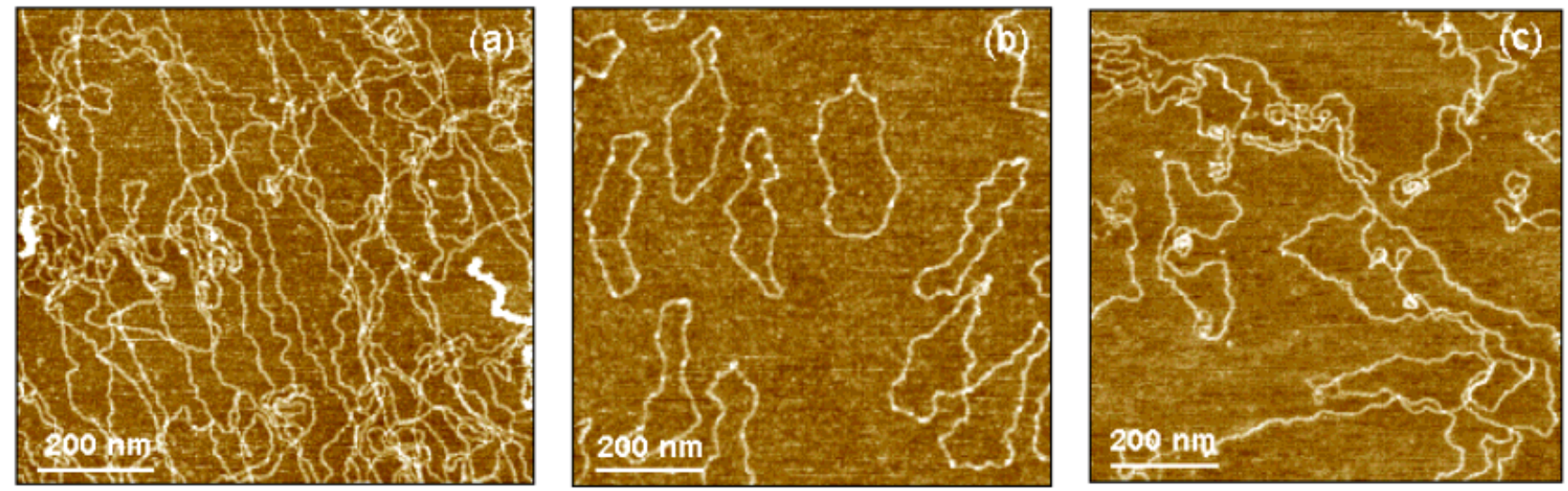

Fig. 1. Topview AFM images of different dsDNA topologies on amino-terminated mica (vertical color scale equals to $3 \mathrm{~nm}$ ) taken in AFM tapping mode under ambient conditions. (a) linear $\lambda$-DNA (with $48.5 \mathrm{kbp}$ ), (b) non-twisted circular DNA plasmids (vector with $3.2 \mathrm{kbp}$ ) and (c) circular supercoiled DNA with twists and writhes due to internal supercoiling (supercoiled DNA ladder 2-16 kbp). The measured width of dsDNA of all different geometrical topologies is typically 3-7 $\mathrm{nm}$ and affected by the tip geometry. The molecular height is typically around $1 \mathrm{~nm}$.

\section{Results and Discussion}

AFM allows a fast characterization of DNA structural properties. In Fig. 1 (a), linear double stranded (ds) $\lambda$-DNA, (b) circular dsDNA plasmids (pGEM), and (c) supercoiled dsDNA plasmids with twists and writhes are shown. All DNA molecules exhibit a width of typically 3-7 nm which is more than the theoretical diameter of $2 \mathrm{~nm}$ and is governed by tip convolution effects [25]. The differences between linear, circular, and circular DNA with supercoiled properties can easily be distinguished and, furthermore, a direct judgement of molecular uniformity and integrity as well as molecular sizing can be made.

In recombinant DNA technology, DNA molecules are cut and religated with enzymes at sequence specific sites. One example is Hae III, a restriction endonuclease, that recognizes dsDNA at the sequence GGCC and cuts the molecule in two pieces within milliseconds. This process is fast, highly efficient, and relies on a specific recognition event between the enzyme (Hae III) and the biopolymer (DNA). Thereby, the molecular complex is believed to undergo local conformational changes due to electrostatic interactions leading to DNA twisting and bending. This is verified by AFM experiments (Fig. 2) which clearly show a distinct local bending of about $90 \pm 20$ degrees in the dsDNA molecular complexes at the recognition site [26]. A bending induced by external forces (e.g. hydrodynamic drag) during surface immobilization is not very likely, since the complexes exhibit identical local bending but always show random angular orientations as demonstrated for two complexes in Fig. 2. The observation of this enzyme-induced conformational change can only be realized by using either mutated suicide restriction enzymes [27] or a non-optimized reaction environment. Here we made use of the combination of an adequate surface immobilization technique and a reaction environment with a reduced temperature of 4 _ C (10 mM TRIS-HCl, $10 \mathrm{mM} \mathrm{MgCl}_{2}, 50$ $\mathrm{mM} \mathrm{NaCl}, 1 \mathrm{mM}$ DTE, $\mathrm{pH}$ 7.5) in order to freeze this process on a surface. It is worth noting, that upon (gaussian) fitting the overall enzyme shape, these experiments generally allow localisation of genetic sequences on individual DNA molecules on a surface with a resolution of about $1 \mathrm{~nm}$.

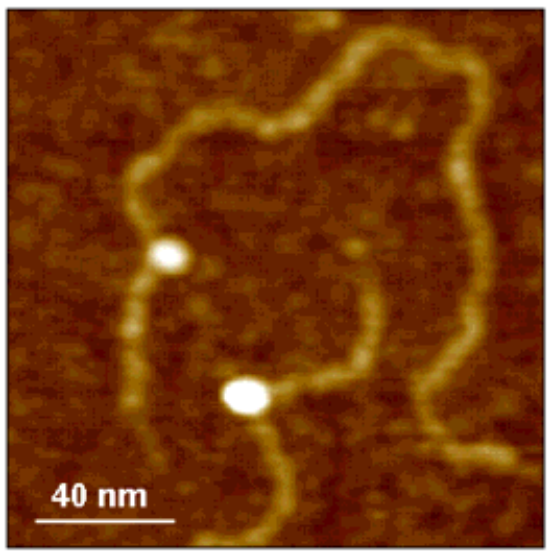

Fig. 2. AFM image (topview) of Hae III-digested $\lambda$-DNA showing two single DNA-restriction enzyme complexes taken in AFM tapping mode under ambient conditions (vertical color scale equals to $4 \mathrm{~nm}$ ). It is apparent that the recognition process induces a local change in the conformation of the DNA by bending it by about 90 degrees.

Not only does AFM offer the possibility of observing individual native biomolecules but it can also be used as a tool to work with molecular precision on the nanometer scale [28]. We used the sharp tip of the AFM cantilever to locally dissect a dsDNA molecule (Fig. 3). Normally, the 
interaction between the sensing AFM tip and the imaged molecule is minimized in order to avoid a mechanical manipulation (displacing or changing) of the immobilized molecules. However, upon increasing locally the force on the DNA molecule and repetitively scanning at a defined position perpendicularly over the molecule, we were able to dissect an individual molecule at a predefined position, simulating the function of a restriction endonuclease. The nanomechanical manipulation was verified by reducing the interaction to its original value and reimaging the dissected molecule within its unaltered surrounding.

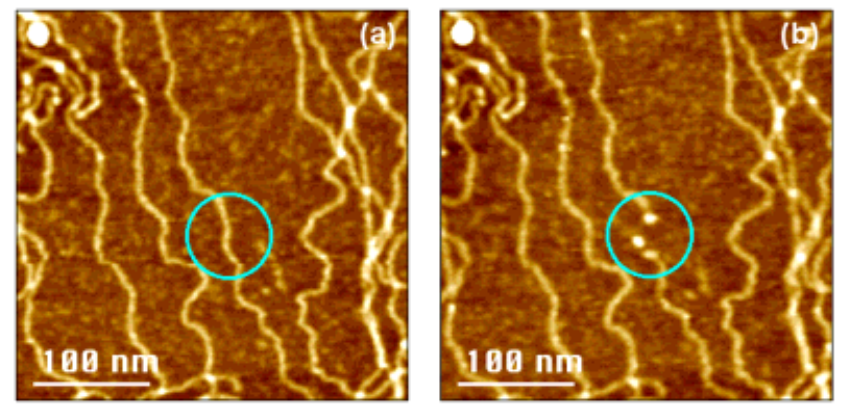

Fig. 3. Two topview AFM images of $\lambda$-DNA in AFM tapping mode under ambient conditions (vertical color scale equals to $3 \mathrm{~nm}$ ) (a) before and (b) after mechanically cutting a dsDNA strand in the center of the image (circle) with the AFM cantilever tip mechanically in two pieces. This controlled molecular manipulation was realized by locally increasing the force interaction between AFM tip and DNA molecule and repetitively scanning perpendicularly over the molecule at a defined position.

Another way to probe individual molecules by AFM are force spectroscopy experiments, where single molecules are picked up and mechanically stretched by an AFM cantilever [12 - 21]. The arbitrariliy chosen molecule is immobilized with one end to the surface and adheres with its other end to the AFM cantilever tip. Upon retracting the cantilever tip from the surface, the molecule is more and more stretched and its mechanical properties under this growing external force can be probed with a very high force sensitivity in the piconewton range $\left(\mathrm{pN}=10^{-12} \mathrm{~N}\right)$. It is interesting to note that these experiments can probe extreme conformational states of individual molecules that are not occupied in "normal" thermodynamic environments. However, it is believed that these "high-force" states play a role in specific binding events where molecules or molecular complexes are locally unwound, twisted, or undergo structural conformation changes as for example in enzymatic processes mentioned above.

Such a force spectroscopic AFM experiment is shown in Fig. 4a. The force response of a synthetic DNA system of pyridine bases (poly(dG-dC)) was measured against its molecular extension in TRIS-HCl buffer at $\mathrm{pH}$ 8.3. The DNA molecule exhibits the non-linear force response of a chainlike biopolymer and additional specific structural transitions
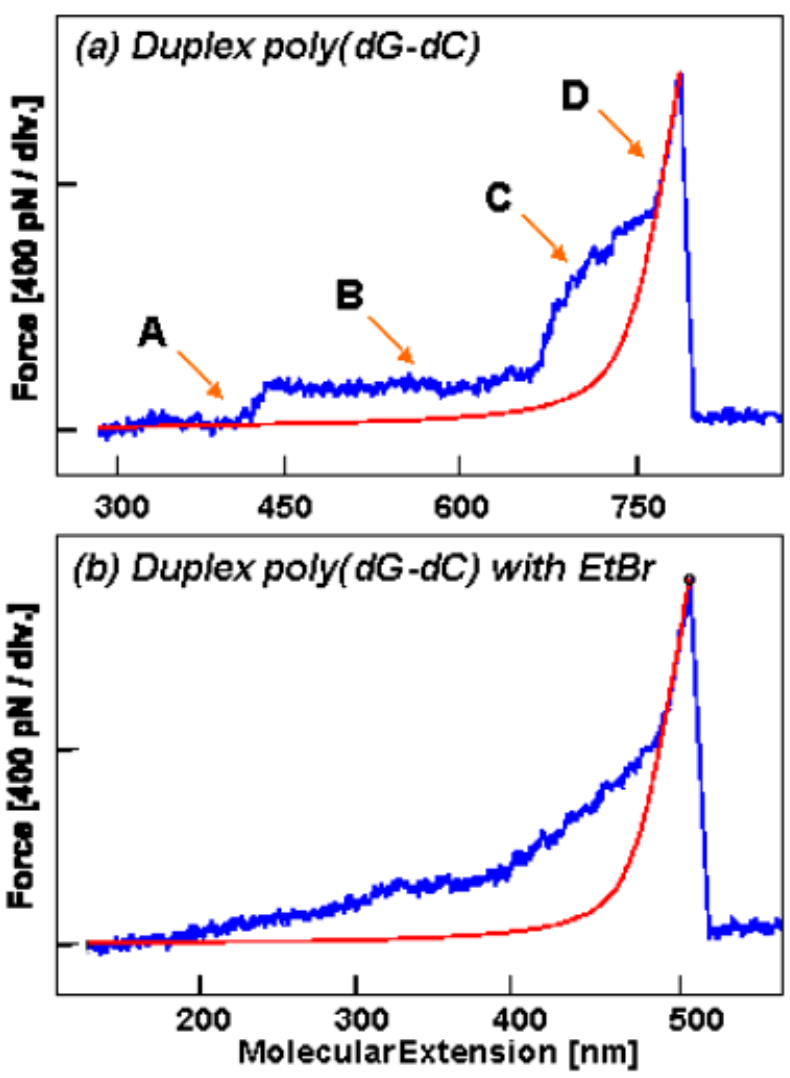

Fig. 4. AFM force spectroscopy experiments with DNA (a) in TRIS buffer and (b) in the presence of a DNA intercalator ethidium bromide (EtBr) (TRIS). The force vs. molecular extension relation reflects the molecular elasticity of dsDNA at small forces $(A)$, the plateau at 65-70 $\mathrm{pN}$ reminescent of the structural transition from $B$ - to $S$-form $(B)$, the mechanical melting of the dsDNA around $300 \mathrm{pN}$ to form ssDNA (C), and the molecular elasticity of ssDNA (D). Upon injecting EtBr, the DNA elasticity immediately changes, suggesting a very fast binding of EtBr to DNA. For details see text. The red curves are elasticity fits to the high-force ssDNA elasticity according to the modified freely jointed chain model (FJC) with (a) $L=778 \mathrm{~nm}, b=0.8 \mathrm{~nm}$ and $S \sim$ $800 \mathrm{pN}(b) L=462 \mathrm{~nm}, b=0.8 \mathrm{~nm}$ and $S \sim 800 \mathrm{pN}$, where $L, b$ and $S$ denote contour length, Kuhn segment length and molecular stretch modulus, respectively.

due to molecular overstretching. The mechanical response of the molecule reflects the internal degrees of freedom of a complex double-stranded helix-type molecule and was observed earlier in experiments with optical microfibers [9], optical tweezers [5], magnetic beads [7] and AFM [20]. The force plateau around $65-70 \mathrm{pN}$ is attributed to a structural transition of DNA from the B-form to the stretched S-form and is verified by a numerical simulation [29]. The second transition around $300 \mathrm{pN}$ is attributed to the mechanical denaturation of dsDNA ending at high forces with a singlestranded (ss) molecular chain [20]. It is worth noting that the biopolymer is able to resist forces up to $0.8 \mathrm{nN}$. The red 
curve is the theoretical fit based on the modified freely jointed chain model (FJC) describing polymer elasticity with an overall molecular contour length of $778 \mathrm{~nm}$, a Kuhn segment length of $0.8 \mathrm{~nm}$, and a molecular stretch modulus of $\sim 800 \mathrm{pN}$ to fit the single-stranded part of the forceextension relation [5]. It is well known that the given elastic parameters depend not only on the nature of the biopolymer itself but also on its local environment $(\mathrm{pH}$, ionic concentration) [5]. Beside protein-DNA binding, which plays an important role in DNA transcription or chromatin organisation, there exist other DNA binding processes, for example involving small molecules that intercalate between the DNA bases or recognize the minor groove structure of the double helix. These binding processes are often utilized to stain and detect DNA or are known to be of mutagenic or oncological relevance. Since binding of small molecules to DNA should also change the corresponding molecular mechanics of the biopolymer [9], we investigated the binding of ethidium bromide (EtBr), a well-established intercalating fluorescence marker [30], by AFM force spectroscopy. As shown in Fig. 4b, the mechanical signature of the probed dsDNA changes dramatically upon interaction with $\mathrm{EtBr}$. Most clearly, the pronounced plateau reflecting the structural transition from B-DNA to S-DNA vanishes. Comparing the two force responses in Fig. 4a and Fig. $4 \mathrm{~b}, \mathrm{EtBr}$ intercalation leads to a faster and linear increase of the force response at small extensions $(<60$ $\mathrm{pN}$ ) reflecting a more flexible double stranded biopolymer which exerts higher entropic forces on the AFM cantilever. Whether this increased biopolymer flexibility is the result of electrostatic charge effects (e.g. charge screening) or other phenomena will be further investigated and verified by molecular mechanics calculations in the future. Other DNA binders (intercalators, minor groove binders, ...) with therapeutic function, e.g. in oncology, are currently being investigated and will be published elsewhere [31].

Concerning future application of this technique, we would like to emphasize that the drastic change in DNA mechanics could be employed in a single molecule DNA biosensor transducer, which transfers DNA affinity into a highly sensitive mechanistic non-fluorescent signal.

\section{Conclusions and Perspectives}

AFM combines high spatial resolution with piconewton force sensitivity, allowing observation and manipulation of individual molecules. This opens new perspectives for biophysical experiments with single molecules, either in monitoring molecular binding phenomena or in characterizing their corresponding mechanical properties.

We characterized DNA systems with different topologies and demonstrated the possibility to visualize enzyme binding to DNA. Manipulation of single DNA molecules was presented by using the sharp tip of an AFM cantilever to cut a distinct single DNA in two pieces. Furthermore, AFM force spectroscopy of single DNA molecules unveiled structural transitions in response to an applied external force, reflecting the structural property of the double helix. This allowed monitoring of specific interaction and binding phenomena of small intercalator molecules with single DNA strands, using a mechanical, non-fluorescent detection scheme.

In conclusion, several advantages of single molecule experiments with AFM have to be mentioned. First, it is possible to probe molecular properties or configurations that are averaged out in ensemble experiments, and second, single molecules can be manipulated in order to investigate molecular conformations which are normally not occupied in thermodynamic equilibrium. In addition, a very pragmatic and simple reason to probe single molecules is that only minor quantities of biomolecules are needed. This could be especially important for experiments investigating rare and expensive samples or where a fast and highly parallel screening is needed. In the future, even higher resolution in space, time and force will allow much more insight into dynamic processes on a molecular scale. This will impact the fundamental identification of important underlying molecular mechanisms and stimulate new visions and strategies in molecular pharmacology, lead finding for drug identification, and also for biomolecular and medical nanotechnology.

Acknowledgements This work was realized within Novartis Services AG in collaboration with the Friedrich-MiescherInstitute (FMI) in Basel, Switzerland. The authors would like to thank Fritz Thommen, Hans-Joachim Güntherodt, Max Burger, and Peter Grütter for their support. Furthermore, macro programming support from Karl Kaufmann and discussions with Christoph Wanke are gratefully acknowledged.

\section{References}

[1] Gimzewski, J.K., Joachim, Ch., Science 283 (1999) 1683-1688.

[2] Guckenberger, R., Heim, M., Cevc, G., Knapp, H.F.,Wiegräbe, W., Hillebrand, A., Science 266 (1994) 1538-1540.

[3] Yanagida, M., Hiraoka, Y., Katsura, I., Cold Spring Harbor Symp. Quant. Biol. 47 (1983) 177.

[4] Wennmalm, S., Edman, L., Rigler, R., Proc. Natl. Acad. Sci. 94 (1997) 10641-10646.

[5] Smith, S.B., Cui, Y., Bustamante, C., Science 271 (1996) 795-799.

[6] Wang, D.W., Yin, H., Landick, R., Gelles, J., Block, S.M., Biophys. J. 71 (1997) 1335-1346.

[7] Strick, T.R., Allemand, J.F., Bensimon, D., Bensimon, A., Croquette, V., Science 271 (1996) 1835-1837. 
[8] Smith, S.B., Finzi, L., Bustamante, C., Science 258 (1992) 1122-1126.

[9] Cluzel, Ph., Lebrun, A., Heller, Ch., Lavery, R., Viovy, J.L., Chatenay, D., Caron, F., Science 271 (1996) 792794.

[10] Fink, H.-W., Schönenberger, Ch., Science 398 (1999) 407-410.

[11] Hansma, H.G., Sinsheimer, R.L., Li, M.-Q., Hansma, P.K., Nucleic Acids Res. 20 (1991) 3585-3590.

[12] Florin, E.-L., Moy, V.T., Gaub, H.E., Science 264 (1994) 415-417.

[13] Dammer, U., Hegner, M., Anselmetti, D., Wagner, P., Dreier, M., Huber, W., Güntherodt, H.-J., Biophys. J. 70 (1996) 2437-2441.

[14] Hinterdorfer, P., Baumgartner, W., Gruber, H.J., Schilcher, K, Schindler, H., Proc. Natl. Acad. Sci. 93 (1996) 3477-3481.

[15] Dammer, U., Popescu, O., Wagner, P., Anselmetti, D., Güntherodt, H.-J., Misevic, G.N., Science 267 (1995) 1173-1175.

[16] Lee, G.U., Chrisey, L.A., Colton R.J., Science 266 (1994) 771-773.

[17] Strunz, T., Oroszlan, K., Schäfer, R., Güntherodt, H.-J., Proc. Natl. Acad. Sci. 96 (1999) 11277-11282.

[18] Fritz, J., Kolbinger, F., Katopodis, A., Anselmetti, D., Proc. Natl. Acad. Sci. 95 (1998) 12283-12288.

[19] Rief, M., Oesterhelt, F., Fernandez, J.M., Gaub, H.E., Science 276 (1997) 1109-1112.
[20] Rief, M., Clausen-Schaumann, H., Gaub, H.E., Nature Struc. Biol. 6 (1999) 346-349.

[21] Rief, M., Gautel, M., Oesterhelt, F., Fernandez, J.M., Gaub, H.E., Science 276 (1997) 308-312.

[22] Lyubchenko, Y., Shlyakhtenko, L., Harrington, R., Oden, P., Lindsay, S., Proc. Natl. Acad. Sci. 90 (1993) 2137-2140.

[23] Neumeister, J.M., Ducker, W.A., Rev. Sci. Instr. 65 (1994) 2527-2531.

[24] Hutter, J.L., Bechhofer, J., Rev. Sci. Instr. 64 (1993) 1868-1873.

[25] Thundat, T., Zheng, X.-Z., Sharp, S.L., Allison, D.P., Warmack, R.J., Joy, D.C., Ferrell, T.L., Scanning. Microsc. 6 (1992) 903-910.

[26] Bustamante, C., Rivetti, C., Annu. Rev. Biophys. Biomol. Struct. 25 (1996) 395-429.

[27] Allison, D.P., Kerper, P.S., Doktycz, M.J., Spain, J.A., Modrich, P., Larimer, F.W., Thundat, T., Warmack, R.J., Proc. Natl. Acad. Sci. 93 (1996) 8826-8829.

[28] Hansma, H., Vesenka, J., Siegrist, C., Keldermann, G., Morret, H., Sinsheimer, R.L., Elings, V., Bustamante, C., Hansma, P.K., Science 256 (1992) 1180-1184.

[29] Lebrun, A., Lavery, R., J. of Biomolec. Struc. \& Dynamics 16 (1998) 593-604

[30] Reinhardt, C.G., Krugh, T.R., Biochemistry 17 (1978) 4845

[31] Smith B., Anselmetti, D., in preparation 